University of Nebraska - Lincoln

DigitalCommons@University of Nebraska - Lincoln

Timothy J. Gay Publications

Research Papers in Physics and Astronomy

May 1988

\title{
Projectile-charge dependence of ejected-electron spectra
}

V. D. Irby

University of Missouri-Rolla, Rolla, Missouri

Timothy J. Gay

University of Nebraska - Lincoln, tgay1@unl.edu

J. Wm. Edwards

University of Missouri-Rolla, Rolla, Missouri

E. B. Hale

University of Missouri-Rolla, Rolla, Missouri

M. L. McKenzie

University of Missouri-Rolla, Rolla, Missouri

See next page for additional authors

Follow this and additional works at: https://digitalcommons.unl.edu/physicsgay

Part of the Physics Commons

Irby, V. D.; Gay, Timothy J. ; Edwards, J. Wm.; Hale, E. B.; McKenzie, M. L.; and Olson, R. E., "Projectilecharge dependence of ejected-electron spectra" (1988). Timothy J. Gay Publications. 15.

https://digitalcommons.unl.edu/physicsgay/15

This Article is brought to you for free and open access by the Research Papers in Physics and Astronomy at DigitalCommons@University of Nebraska - Lincoln. It has been accepted for inclusion in Timothy J. Gay Publications by an authorized administrator of DigitalCommons@University of Nebraska - Lincoln. 


\section{Authors}

V. D. Irby, Timothy J. Gay, J. Wm. Edwards, E. B. Hale, M. L. McKenzie, and R. E. Olson 
Phys. Rev. A 37, 3612 - 3614 (1988)

[Issue 9 - May 1988]

\title{
Projectile-charge dependence of ejected-electron spectra
}

\author{
V. D. Irby, T. J. Gay, J. Wm. Edwards, E. B. Hale, M. L. McKenzie, \\ and R. E. Olson \\ Laboratory for Atomic and Molecular Research and Physics Department, \\ University of Missouri-Rolla, Rolla, Missouri 65401
}

\begin{abstract}
We have studied $\mathrm{H}^{+}+\mathrm{He}$ and ${ }^{3} \mathrm{He}^{++}+\mathrm{He}$ ionizing collisions at intermediate energies (60 to $120 \mathrm{keV} / \mathrm{amu}$ ) and have observed a significant shift in the velocity position of the maxima in forward-ejected electron spectra when changing projectiles from protons to $\alpha$ particles. In ionizing collisions, a large portion of the ejected electrons are stranded near the transitory equiforce, or saddle point, position between the target ion and receding projectile. The shift in the electron spectra maxima to smaller velocities, due to changing projectiles from protons to $\alpha$ particles, is consistent with the shift in the saddle-point velocity when the projectile charge is increased.
\end{abstract}

CC1988 The American Physical Society

URL: $\underline{\text { http://link.aps.org/abstract/PRA/v37/p3612 }}$

DOI: $10.1103 /$ PhysRevA.37.3612

PACS: $34.50 . \mathrm{Fa}$ 


\title{
Rapid Communications
}

The Rapid Communications section is intended for the accelerated publication of important new results. Since manuscripts submitted to this section are given priority treatment both in the editorial office and in production, authors should explain in their submittal letter why the work justifies this special handling. A Rapid Communication should be no longer than 31/2 printed pages and must be accompanied by an abstract. Page proofs are sent to authors, but, because of the accelerated schedule, publication is not delayed for receipt of corrections unless requested by the author or noted by the editor.

\section{Projectile-charge dependence of ejected-electron spectra}

\author{
V. D. Irby, T. J. Gay, J. Wm. Edwards, E. B. Hale, M. L. McKenzie, and R. E. Olson \\ Laboratory for Atomic and Molecular Research and Physics Department, University of Missouri-Rolla,
}

Rolla, Missouri 65401

(Received 4 January 1988)

\begin{abstract}
We have studied $\mathrm{H}^{+}+\mathrm{He}$ and ${ }^{3} \mathrm{He}^{2+}+\mathrm{He}$ ionizing collisions at intermediate energies $(60$ to $120 \mathrm{keV} / \mathrm{amu}$ ) and have observed a significant shift in the velocity position of the maxima in forward-ejected electron spectra when changing projectiles from protons to $\alpha$ particles. In ionizing collisions, a large portion of the ejected electrons are stranded near the transitory equiforce, or saddle point, position between the target ion and receding projectile. The shift in the electron spectra maxima to smaller velocities, due to changing projectiles from protons to $\alpha$ particles, is consistent with the shift in the saddle-point velocity when the projectile charge is increased.
\end{abstract}

In a recent paper, ${ }^{1}$ we presented calculations and measurements of $\mathrm{H}^{+}+\mathrm{He}$ ionizing collisions and found that ejected electrons traveling roughly midway between the two post-collision centers dominated the forward-ejection electron velocity spectra at intermediate projectile energies. This was attributed to the stranding of electrons on the transitory Coulomb equiforce, or saddle point, region between the two post-collision centers. This phenomenon can be easily visualized classically.

In this paper, we examine the effect of increasing the projectile charge on the relative position of the peak in electron velocity spectra at a forward-ejection angle of $17^{\circ}$. Specifically, we have investigated ionization in collisions of protons and $\alpha$ particles with helium at $E=60-120 \mathrm{keV} / \mathrm{amu}$ :

$$
\begin{aligned}
& \mathrm{H}^{+}+\mathrm{He} \rightarrow \mathrm{H}^{+}+\mathrm{He}^{+}+e^{-}, \\
& \mathrm{He}^{2+}+\mathrm{He} \rightarrow \mathrm{He}^{2+}+\mathrm{He}^{+}+e^{-} .
\end{aligned}
$$

(At these energies, double electron removal is essentially negligible, ${ }^{2}$ although we do not discriminate against it experimentally.) We present calculations and experimental data which further establish the importance of the saddle-point mechanism by illustrating the shift in electron velocity spectra due to an increase of projectile charge.

Figure 1 compares electronic potential plots for the two systems. In each case the target ion is at rest and the projectile is traveling with a speed $v_{p}$. When the charge of the projectile is increased, its Coulomb well becomes deeper and the traveling equiforce position is shifted toward the lesser charge. The velocity of the equiforce, or saddle-point location can be shown to be

$$
v_{s}=\frac{v_{p}}{1+\left(q_{p} / q_{T}\right)^{1 / 2}},
$$

where $q_{p}$ and $q_{T}$ are the final charges of the projectile and target, respectively. When the projectile is changed from

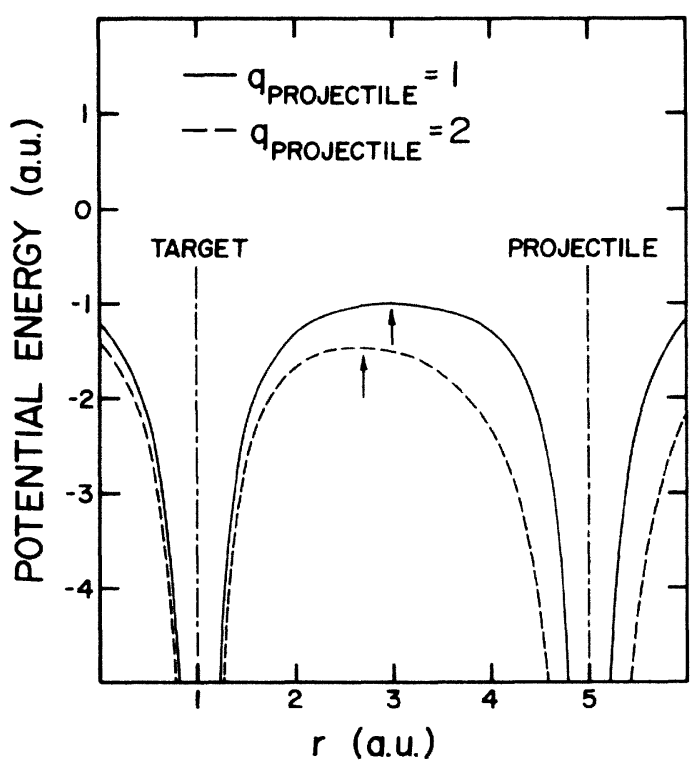

FIG. 1. Potential energy plots for $\phi=-\left(q_{T} /|1-r|\right)$ $-\left(q_{p} /|5-r|\right)$, where $q_{r}$ and $q_{p}$ are the final target and projectile charges, respectively. The target is placed at $r=1$ for convenience. 
$\mathrm{H}^{+}$to $\mathrm{He}^{2+}$, the velocity of the saddle point changes from $v_{s}=0.50 v_{p}$ to $v_{s}=0.41 v_{p}$. Since a large portion of the ejected electrons are stranded near the saddle position in ionizing collisions, ${ }^{1}$ one would expect to observe an "inward" shift in the peak of the ejected-electron velocity spectrum when comparing $\alpha$ particles as incident projectiles to protons. This behavior is different than our initial naive expectation that, due to the higher charge on the $\alpha$ particle, the electrons would feel a stronger pull which would tend to shift the peak to a higher velocity.

To search for a saddle-point velocity shift with a helium-atom target, we have made comparisons of ejected-electron spectra for both protons and $\alpha$ particles with the same projectile velocities. Much of the experimental apparatus has been previously described. ${ }^{1,3,4} \mathrm{We}$ choose to use ${ }^{3} \mathrm{He}^{2+}$ instead of ${ }^{4} \mathrm{He}^{2+}$, due to the difficulty of electromagnetically separating ${ }^{4} \mathrm{He}^{2+}$ from $\mathrm{H}_{2}{ }^{+}$, which is abundant in the source. In order to produce adequate currents of ${ }^{3} \mathrm{He}^{2+}$, we have switched to using a microwave-ion source instead of our usual Colutron source (the maximum ${ }^{3} \mathrm{He}^{2+}$ beam current obtained with the Colutron was about $0.5 \mathrm{nA}$ ). The microwave source, similar to that developed by Walther, Leung, and Kunk$\mathrm{el},{ }^{5}$ consisted of an 8-mm i.d. glass tube which was inserted inside a quarter-wave stub tuner. A source gas mixture of four parts ${ }^{3} \mathrm{He}$ and one part $\mathrm{Ar}$ resulted in a maximum beam current of $25 \mathrm{nA}$, with typical currents of 5 to $6 \mathrm{nA}$ measured at the entrance to our target chamber.

The ion beam crossed an effusive helium target in single collision conditions. The electron-energy analyzer was of the parallel-plate type, and could be used at angles of $0^{\circ}$, by virtue of an aperture in the analyzer back plate, or at angles between $17^{\circ}$ and $120^{\circ}$, a range dictated by analyzer and chamber geometry. The retardation and acceleration grids discussed in Ref. 3 were not used in this work. Magnetic fields in the interaction volume were reduced to less than $10.0 \mathrm{mG}$ by Helmholtz coils and mumetal shields. Possible effects due to contact potentials, electron absorption in background gas, and a neutral beam fraction were studied and shown to be negligible.

In Fig. 2, we compare typical spectra for $\mathrm{H}^{+}$and ${ }^{3} \mathrm{He}^{2+}$, both with the same incident velocities. An $x-y$ recorder was used to collect the data with the $x$ axis driven by the changing voltage across the analyzer plates, and the $y$ axis driven by the output of a rate meter. The signal-to-noise ratio was smaller for the $\alpha$ particles due to lower incident beam currents. The detector efficiency function was determined by making a calibration run involving $\mathrm{H}^{+}+\mathrm{He}$ with an incident proton energy of 100 $\mathrm{keV}$, and an electron-ejection angle of $30^{\circ}$. This spectrum was then compared to the data of Rudd, Toburen, and Stolterfoht, ${ }^{6}$ and the relative detection efficiency versus electron energy was obtained by dividing Rudd's data by our unnormalized cross sections obtained from the $x-y$ plots. Calibration runs were made both before and after experimental data were taken and the detector efficiency was observed to remain constant. Beam current, chamber pressure, and rate-meter gain were recorded, and using the detector efficiency function, the relative doublydifferential cross sections were obtained by normalizing to the calibration run. Absolute cross sections were then

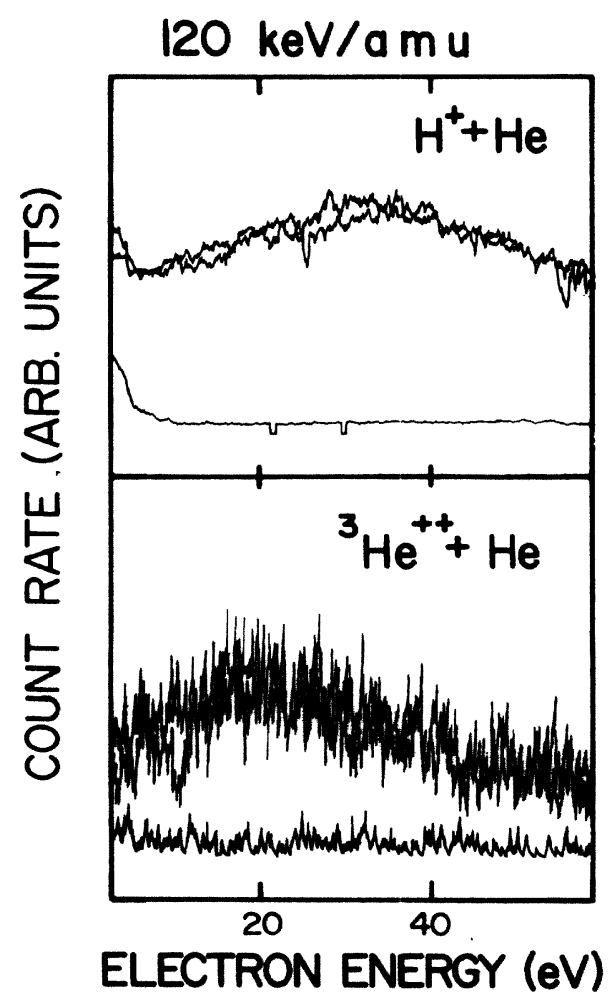

FIG. 2. Electron spectra taken for incident projectile energies of $120 \mathrm{keV} / \mathrm{amu}$ and an ejection angle of $17^{\circ}$. The electron energy scan is from 4 to $60 \mathrm{eV}$ for both protons and $a$ particles. The upper curves for each spectrum were taken with gas-in (chamber pressure $=2 \times 10^{-5}$ Torr uncorrected for helium); the lower curves are the gas-out background scans (chamber pressure $=3 \times 10^{-6}$ Torr).

computed by normalizing the analyzed data to the absolute cross sections reported by Rudd et al. ${ }^{6}$ for $50 \mathrm{eV}$ electrons ejected at $30^{\circ}$ by $100-\mathrm{keV}$ incident protons.

As can be seen in Figs. 2-4, there exists a significant "inward" shift of the peak in the electron velocity spectra at 60,100 , and $120 \mathrm{keV} / \mathrm{amu}$, when the charge of the projectile is changed from 1 to 2 . The shift in the electron velocity spectra corresponds to the velocity shift of the saddle point when the projectile charge is increased. We note that while the velocity of the saddle point is $0.41 v_{p}$ and $0.50 v_{p}$ for $\alpha$ particles and proton projectiles, respectively, all these data exhibit peaks at $v_{e} / v_{p}>0.5$. The peak position is a function of projectile velocity and electron emission angle as well as the projectile charge. As the projectile velocity is increased, the relative importance of "direct," or target-centered ionization increases, and the peak shifts to a lower relative velocity (for example, the $17^{\circ}$ electron spectrum peaks at $v_{e} / v_{p} \simeq 0.35$ for $200 \mathrm{keV}$ protons in Ref. 1). The peak position does not appear to be sensitive to electron emission angle below about $10^{\circ}, 4,7$ but at larger angles, direct ionization begins to dominate the saddle-point contribution and the peak again shifts to lower velocities. Thus, while classical arguments based on the simple geometry of the potential saddle point are qualitatively correct, quantitative predictions must include the dynamical effects of projectile and electron velocity.

In order to include these effects quantitatively, we have 


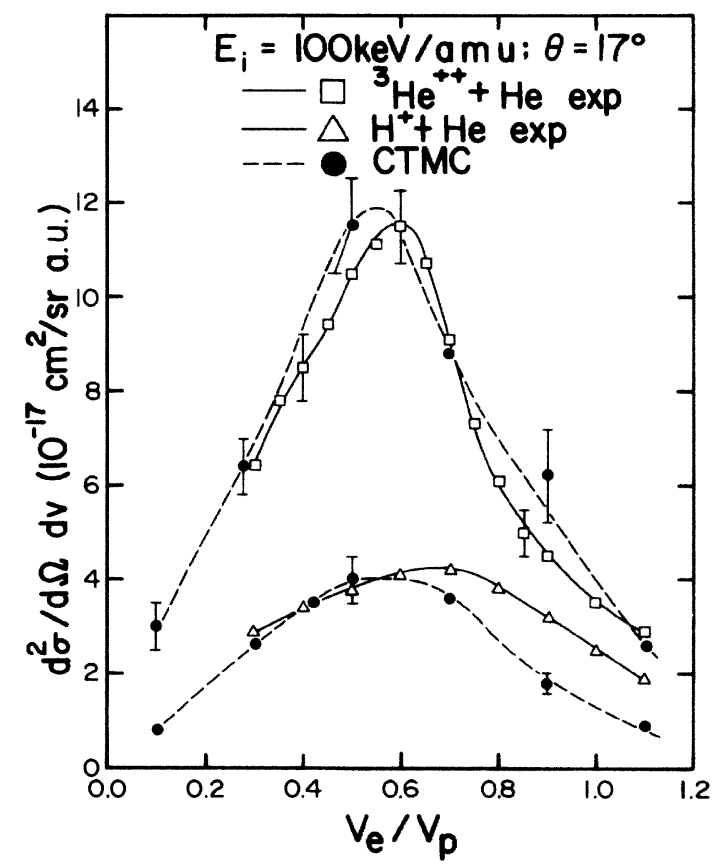

FIG. 3. Doubly-differential cross sections for electron production at $100 \mathrm{keV} / \mathrm{amu}$ plotted in electron velocity space normalized to the projectile velocity. Open triangles are the proton data, and open squares are the $a$ data. CTMC results are indicated with solid circles. Relative error for the proton data is less than 5\%. Relative error for the $\alpha$ data is indicated. Absolute error resulting from normalization to the data of Rudd is about $25 \%$. The dashed and solid lines serve to guide the eye.

performed classical trajectory Monte Carlo (CTMC) calculations $^{8,9}$ for reactions (1) and (2) at $100 \mathrm{keV} / \mathrm{amu}$. The independent electron model ${ }^{10}$ was used where the single-ionization transition probability is given by

$$
P(b)=2 p_{i}(b)\left[1-p_{i}(b)-p_{c}(b)\right] .
$$

In Eq. (4), the ionization and electron-capture probabilities $p_{i}(b)$ and $p_{c}(b)$ are determined within the singleelectron framework of the CTMC method. For the He atom, we used a $Z_{\text {eff }}=1.6875$ and an ionization potential of $24.58 \mathrm{eV}$. The effects of electron capture are explicitly included in the calculations. It should be noted that for the $\mathrm{He}^{2+}+\mathrm{He}$ system, the coupling to ionization and electron-capture channels is quite strong, so that the $2\left[1-p_{i}(b)-p_{c}(b)\right]$ factor of Eq. (4) varies from 0.4 at small impact parameters to 2.0 for the large impact parameter collisions.

We find good agreement between theory and experi-

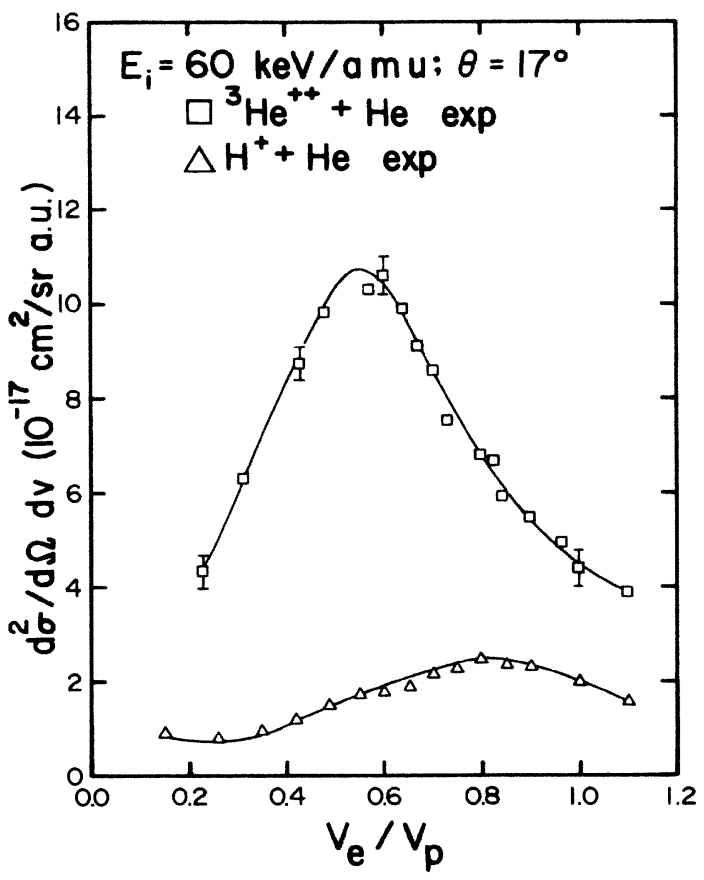

FIG. 4. Doubly-differential cross sections for electron production at $60 \mathrm{keV} / \mathrm{amu}$. Relative error in the $\alpha$-particle data is shown.

ment (Fig. 3). The classical calculations tend to underestimate the peak positions, but there is excellent agreement in the magnitude of the doubly-differential cross sections.

In conclusion, we would like to point out the importance of making measurements at nonzero ejection angles. At $0^{\circ}$, the electron velocity spectra are dominated by electrons traveling at the speed of the projectile $v_{p}$. These electrons originate from "charge capture to the continuum" (CTC) by the projectile and "wash out" the saddlepoint electrons. In order to unambiguously observe the major ionization mechanisms, one has to make measurements at larger ejection angles where the CTC electrons no longer complicate the spectra. We have observed projectile charge-dependent spectra shifts at angles as high as $30^{\circ}$. These shifts can be easily understood classically and are an inherent signature of the saddle-point mechanism.

We would like to thank Dieter Schneider and Gordon Berry for the loan of the electron spectrometer and several helpful discussions. This work was supported by the U.S. Department of Energy-Office of Fusion Energy, and the Physics Division of the National Science Foundation.
${ }^{1}$ R. E. Olson, T. J. Gay, H. G. Berry, E. B. Hale, and V. D. Irby, Phys. Rev. Lett. 59, 36 (1987).

${ }^{2}$ R. D. DuBois, Phys. Rev. A 36, 2585 (1987).

${ }^{3}$ P. W. Arcuni, Ph.D. thesis, University of Chicago, 1985 (unpublished); Phys. Rev. A 33, 105 (1986); P. W. Arcuni and D. Schneider, ibid. 36, 3059 (1987). The apparatus described in these references was moved to Rolla for this work.

${ }^{4}$ T. J. Gay, H. G. Berry, E. B. Hale, V. D. Irby, and R. E. Olson, Nucl. Instrum. Methods (to be published).

${ }^{5}$ S. R. Walther, K. N. Leung, and W. B. Kunkel, Rev. Sci.
Instrum. 57, 1531 (1986).

${ }^{6}$ M. E. Rudd, L. H. Toburen, and N. Stolterfoht, At. Data Nucl. Data Tables 18, 413, (1976).

${ }^{7}$ D. K. Gibson and I. D. Reid, J. Phys. B 19, 3256 (1986); Australian Atomic Energy Commission Report No. E608, 1985 (unpublished).

${ }^{8}$ I. C. Percival and D. Richards, Adv. At. Mol. Phys. 11, 1 (1975).

${ }^{9}$ R. E. Olson and A. Salop, Phys. Rev. A 16, 531 (1977).

${ }^{10}$ J. H. McGuire and L. Weaver, Phys. Rev. A 16, 41 (1977). 